\title{
The Analysis of Relationship between Urbanization and Education Level Dynamic Measurement
}

\author{
Yuewen Ren \\ School of Economic, Sichuan University, Chengdu 610000, China \\ 973906264@qq.com
}

\begin{abstract}
This paper constructs a comprehensive evaluation index system of city level panel data of 18 cities and counties of Hainan Province based on 2000-2011, using structural equation model for the comprehensive measure of our country's urbanization level. And on this basis, the quantitative analysis of Hainan province education situation and urbanization level dynamic causal relationship, providing a sentific basis for establishing policies to improve the urbanization level of Hainan province and improving the education condition.
\end{abstract}

Keywords: urbanization level, education condition, structural equation model.

\section{Introduction}

The so-called urbanization means that with the development of social productive forces, the population of a country or region is transferred from the countryside to the city, and the production and life of mankind are transformed from rural to urban areas. It is the process that the city's infrastructure and public service facilities continue to improve, the structure and level of consumption and investment continue to improve, and urban culture and urban values become the main body of the urban areas.

From the perspective of sociological research, education has the function of promoting social mobility, and social stratification theory is the prerequisite of social labor flow, social stratification inspires people through education to obtain professional qualifications, in order to achieve a higher level of movement. Under the context of the rapid progress of urbanization, the strategy of strengthening the country through talents and the strategy of rejuvenating the country through science and education is not only an inevitable requirement of urbanization and economic development, but also a concrete manifestation of social progress. Education and urbanization are interdependent and interdependent. Education is the prerequisite for urban culture and urban values to become the main body, and the level of urbanization also provides the material basis and economic impetus for the existence and development of education, which is the guarantee of education progress and development.

\section{Literature review}

$\mathrm{Xu}$ Chaojun et al. (2007) analyzed the promotion of education to urbanization through four aspects: educational investment, human capital, scientific and technological progress and urban culture. It believed that in the 21st century, the mode of economic operation and the continuous progress of urbanization; China Population and Development Research Center Task Force (2009) elaborated the connotation of the education priority development strategy, combined with the current situation and trend of China's education development, pointed out that the education of all levels in our country entered the coordinated development period which was dominated by the structure optimization, and the educational resources entered the integration period, the urban and rural education development entered the adjustment period of the urbanization of population; Wang Jiating, Cui Fengyu (2010) used the econometric methods to study the relations between the structure of China's population education and the urbanization. It was found that the population education structure is the Granger cause of urbanization in China, and there is a long-term cointegration relationship between different educational level and urbanization level, and the contribution of different educational levels to 
urbanization process is different; Zhang Xinmin (2011) used the relevant panel data of Henan zhongyuan urban agglomeration, northern Henan economic zone, western Henan economic zone and Huanghuai economic zone, and made an empirical study on the impact of urbanization on rural education. The increase of the level of development has a strong effect on the increase of the number of students in rural compulsory education and the increase of the number of rural schools. The increase of urbanization rate and the increase of urban population have the effect of pulling down; Weng Jinghua, Han Yuqi (2012) analyzed the relationship between urbanization and higher education in China, and found that it was basically compatible with the development of urbanization, economic development and the scale of higher education in Wenzhou. There were some structural contradictions and problems in this coordination.

\section{Selection of Urbanization Index and Comprehensive Measure}

\subsection{Selection of Urbanization Index}

According to Chen Yongjun (2010), the urbanization is summarized as follows: first, the speed of economic development has increased significantly; second, the proportion of agricultural activities has decreased; the third is non-agricultural population gradually increased, to build the Hainan Province urbanization level index system (Table 1), including a total of three categories of eight indicators. The data source is the "Hainan Statistical Yearbook".

Table 1 Indicator system of urbanization level of 18 cities and counties in Hainan Province

\begin{tabular}{|c|c|}
\hline $\begin{array}{l}\text { Second order index (potential } \\
\text { variable, } \xi \text { ) }\end{array}$ & Specific indicators (observed variables, $\mathrm{X}$ ) \\
\hline \multirow{2}{*}{ Population Urbanization } & $\begin{array}{l}\mathrm{X}_{1} \text { non-agricultural population in each region accounted for the } \\
\text { proportion of the city's total population }\end{array}$ \\
\hline & $\begin{array}{l}\mathrm{X}_{2} \text { second and third industry practitioners accounted for the } \\
\text { proportion of the city's total population }\end{array}$ \\
\hline \multirow{3}{*}{ Economic urbanization } & $\mathrm{X}_{3}$ per capita income \\
\hline & $\mathrm{X}_{4}$ average per capita GDP \\
\hline & $\mathrm{X}_{5}$ the proportion of industry to GDP \\
\hline \multirow{3}{*}{ Social urbanization } & $\mathrm{X}_{6}$ number of faculty and staff in school(per ten thousand people) \\
\hline & $\mathrm{X}_{7}$ number of doctor(per ten thousand people) \\
\hline & $\mathrm{X}_{8}$ number of hospital bed(per ten thousand people) \\
\hline
\end{tabular}

\subsection{Comprehensive measurement of urbanization level}

In order to avoid the overlapping of information between compound index variables and the weight of human subjective factors, this paper uses structural equation model to measure the urbanization level of 18 cities and counties in Hainan Province from 2000 to 2011, and the data source is Hainan Statistical Yearbook (2001-2012). Before the factor analysis, the data needs to be dimensionless (no effect on the structural equation model) to ensure the accuracy of the data and the scientific nature of the evaluation. The Cronbach's Alpha value is required to be at least 0.6 , which is 0.829 , which is much larger than 0.6 , and it is considered that the consistency reliability is so suitable.

The degree of urbanization is incorporated into the structural equation model, and the urbanization level is measured by LISREL9.1 software. The results are shown in Fig. 1. The results of the fitting index are NFI $=0.903, \mathrm{CFI}=0.904, \mathrm{IFI}=0904, \mathrm{NNFI}=0.841, \mathrm{RFI}=0.839, \mathrm{GFI}=0.855, \mathrm{RMR}=$ 0.0167, RMSEA = 0.204, where NFI, CFI and IFI have reached the standard of 0.9 Level, NNFI, RFI, GFI, although not reached 0.9 , but are very close, has been acceptable range, all indicators are acceptable, so the pattern fit and index fit better. This paper concludes that since the data comes from "Hainan Statistical Yearbook" (October 2002 16, the State Council approved (State letter [2002] 92) revoked Qiongshan City, located in Qiongshan District, Haikou City, population and economy suddenly increased significantly, so Haikou City, urbanization and economic urbanization indicators abnormal phenomenon normal). In order to ensure the originality and objectivity of the data, this paper 
does not use other methods to meet the standard and over-subjective, and that the impact coefficient is true, reliable and scientific.

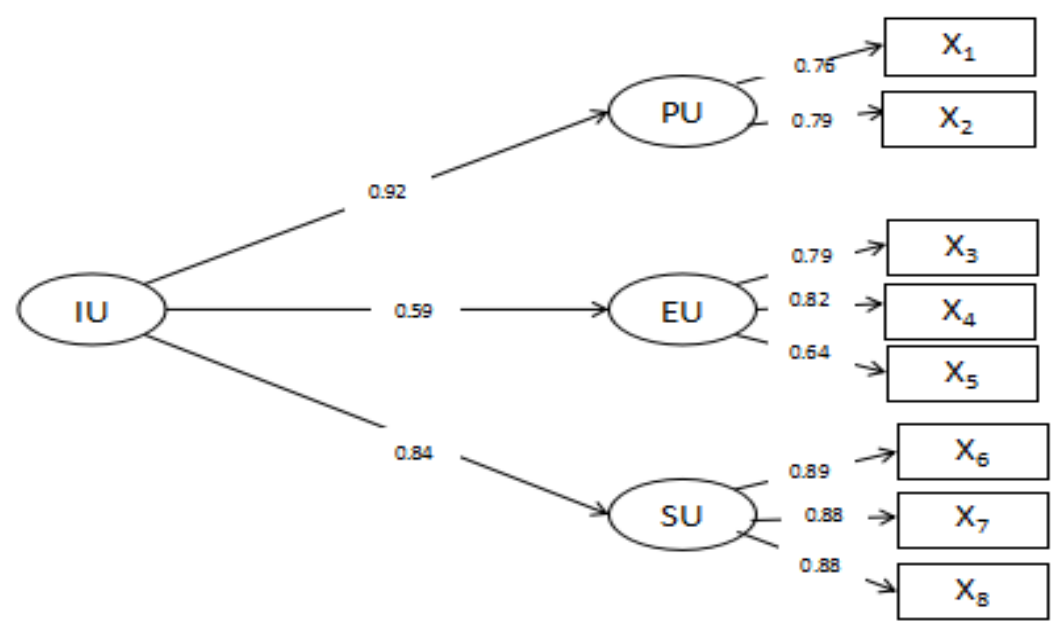

Chi-Square $=240.18, \mathrm{df}=17, \mathrm{P}$-value $=0.000, \mathrm{RMSEA}=0.204$

Fig. 1 Urbanization level indicators Comprehensive measures Analysis results

According to the results of the model fitting (Figure 1, IU represents integrated urbanization, PU represents population urbanization, EU represents economic urbanization, SU represents social urbanization), U1, U2 and $\mathrm{U} 3$ represent the integrated urbanization, population urbanization, economic urbanization and social urbanization respectively. The structural equation of the final comprehensive urbanization level can be expressed as:

$$
\mathrm{IU}=0.4563 \mathrm{PU}+0.2243 \mathrm{EU}+0.3194 \mathrm{SU}
$$

Comparing the average of the comprehensive urbanization levels for the period from 2000 to 2011 ,

$$
\text { that is, } U=\sum_{\mathrm{i}=1}^{12} U_{\text {综合 }} / 12
$$

Where $\mathrm{i}=1,2, \ldots, 12$, ie 2000 - 2011.According to the formulas (1) and (2), the comprehensive urbanization level scores of 18 cities and counties in Hainan Province from 2000 to 2011 are calculated (Table 2).

Table 2 Hainan Province 2000-2011 urbanization level and scoring ranking list

\begin{tabular}{cccccc}
\hline Names & IU & rank & PU & EU & SU \\
\hline Haikou City & 4.223 & 3 & 1.529 & 1.432 & 1.262 \\
Sanya City & 4.426 & 1 & 1.535 & 1.549 & 1.342 \\
Wuzhishan City & 4.309 & 2 & 1.321 & 1.623 & 1.364 \\
Wenchang City & 3.072 & 4 & 1.04 & 0.76 & 1.272 \\
Qionghai City & 2.513 & 6 & 0.8 & 0.999 & 0.714 \\
Wanning City & 2.533 & 5 & 0.86 & 0.935 & 0.738 \\
Ding'an County & 2.2 & 14 & 0.84 & 0.727 & 0.633 \\
Tunchang County & 2.14 & 17 & 0.84 & 0.615 & 0.686 \\
Chengmai County & 2.212 & 13 & 0.872 & 0.643 & 0.697 \\
Lingao County & 2.338 & 10 & 0.859 & 0.847 & 0.633 \\
Danzhou City & 2.217 & 12 & 0.848 & 0.716 & 0.653 \\
Orient City & 2.483 & 7 & 0.849 & 0.953 & 0.682 \\
Ledong County & 2.18 & 15 & 0.695 & 0.79 & 0.695 \\
Qiongzhong County & 2.156 & 16 & 0.826 & 0.507 & 0.823 \\
Baoting County & 2.428 & 9 & 0.96 & 0.506 & 0.963 \\
Lingshui County & 2.235 & 11 & 0.878 & 0.591 & 0.766 \\
Baisha County & 2.089 & 18 & 0.674 & 0.673 & 0.742 \\
Changjiang County & 2.46 & 8 & 0.888 & 0.694 & 0.877 \\
\hline
\end{tabular}




\section{Calculation of Educational Status in Hainan Province}

In view of the availability of the current data, the calculation of the educational situation is also more complicated, as well as the actual situation in China and the constraints of research conditions, this paper uses the weight of the average age of education to calculate the education status. According to the actual statistics of Hainan Province, this paper compares the urban and rural residents' data of urban and urban residents in 18 cities and counties in Hainan Province, The impact of urban civic education) is divided into the following four levels: primary school below, primary school, secondary school (including junior high school and high school) and above high school. The following population, mainly including the illiterate population and the semi-illiterate population (also known as the primary school dropout population), because the relevant statistical yearbook data is missing, the calculation can not be carried out, so the direct withdrawal, this article mainly emphasizes the education population, discarding the number of educated demographics below primary school. The average number of years of education in secondary school and high school is 9 years and 12 years respectively. Let L1 be the number of educated population in primary school, L2 be the number of educated population in primary school and high school, so the average age of education is 6 years. $(9+12) / 2=$ 10.5 years. Let L3 be the number of educated population above high school, including college, undergraduate, master and doctor. As the existing statistics do not subdivide the level of education, this model will ignore the differences between the years of education of undergraduate, undergraduate, masters and $\mathrm{PhDs}$, and the average age of education for those In order to ensure that the number of higher education is 16 years, the statistical methods are as follows: the number of educated population in high school and above urban areas = Number of Senior High School Graduates from the cities and counties in each city and county *(Overall Number of Ordinary Higher Education Institutions / Total Number of High School Graduates). Thus, we can get the urban education in Hainan Province, the calculation formula is as follows:

$$
L=\left(6 L_{1}+10.5 L_{2}+16 L_{3}\right) /\left(L_{1}+L_{2}+L_{3}\right)
$$

Through the calculation, the results of the average years of urban education in Hainan Province from 2000 to 2011 can be obtained (Table 3).

Table 3 Hainan Province from 2000 to 2011 the average years of education statistics

\begin{tabular}{|c|c|c|c|c|c|c|c|c|c|c|c|c|}
\hline Name: & 2000 & 2001 & 2002 & 2003 & 2004 & 2005 & 2006 & 2007 & 2008 & 2009 & 2010 & 2011 \\
\hline Hailegu City & 9.61 & 9.5 & 9.61 & 9.69 & 9. 76 & 9.83 & 9.84 & 10. 18 & 10.04 & 9.41 & 10. 14 & 10.22 \\
\hline Senya City & 8. 6 & 9.6 & 9.74 & 9.96 & 10.06 & 10.15 & 10.4 & 10.57 & 10.77 & 10.57 & 9.91 & 10. 1 \\
\hline Wurhi=her City & 9.05 & 9.53 & 9. 36 & 9.43 & 9.59 & 9.74 & 10.5 & 10.21 & 10.53 & 10. 21 & 10. 75 & 10. 89 \\
\hline Werselheres City & 10.09 & 9. 76 & 9. 74 & 9.86 & 10.02 & 10.09 & 10. 17 & 10.64 & 10.54 & 10.52 & 10.42 & 9.87 \\
\hline Rionghai City & 9.49 & 9.83 & 9.77 & 9.9 & 10 & 10. 13 & 10. 13 & 10. 17 & 10. 31 & 10.23 & 10. 24 & 10.04 \\
\hline Wenring City & 9. 34 & 9.61 & 9.62 & 9.84 & 9.91 & 9.78 & 10.03 & 10.62 & 10. 87 & 10.45 & 10.54 & 9.89 \\
\hline Dirne'an County & 9.8 & 9.87 & 9. 81 & 9.83 & 9.88 & 9.99 & 10. 18 & 10. 11 & 10.43 & 10.42 & 10. 45 & 9. 78 \\
\hline Turchere County & 9. 18 & 9.45 & 9.57 & 9.68 & 9. 86 & 9.99 & 10. 12 & 10.52 & 10. 81 & 10.64 & 10. 35 & 9.95 \\
\hline Cheremein, County & 9.9 & 9.88 & 9.45 & 9.54 & 9.73 & 9.94 & 10. 08 & 11.02 & 10.46 & 10. 68 & 10. 15 & 10. 1 \\
\hline Linese County & 9.66 & 9. 14 & 9.27 & 9. 34 & 9.45 & 9.57 & 9.65 & 10. 19 & 10.21 & 10.07 & 10. 24 & 9.89 \\
\hline Dershore, City & 9.63 & 9. 35 & 9. 33 & 9.18 & 9. 33 & 9.24 & 9. 81 & 10. 19 & 10.29 & 10.58 & 10. 44 & 9. 35 \\
\hline Orient City & 9.45 & 9. 33 & 9.5 & 9.67 & 9.68 & 9.94 & 10. 04 & 10. 32 & 10. 79 & 10.53 & 10. 78 & 9.99 \\
\hline Ledons County & 9.47 & 9. 76 & 9.67 & 9.87 & 10 & 10.05 & 10. 34 & 10. 7 & 10. 83 & 10. 77 & 11. 11 & 10.5 \\
\hline Qiongzhon, County & 9.07 & 9.44 & 9.62 & 9.59 & 9. 81 & 10. 12 & 10.23 & 10.43 & 10.3 & 10. 74 & 10.64 & 9.9 \\
\hline Beoting County & 9.26 & 9.54 & 9.45 & 9.75 & 9.91 & 10.05 & 10. 21 & 10. 19 & 10.66 & 10. 89 & 10. 85 & 9.99 \\
\hline Lineshui, County & 9.5 & 9.45 & 9.55 & 9.65 & 9.79 & 10.01 & 10.07 & 10.53 & 10.52 & 10.47 & 10.45 & 10. 12 \\
\hline Berisho County & 9.74 & 9. 39 & 9.44 & 9.57 & 9. 78 & 9.92 & 10. 18 & 10.03 & 10.22 & 10.55 & 10.3 & 9.91 \\
\hline Chareinisus County & 9. 32 & 8. 93 & 9.29 & 9.4 & 9. 54 & 9.59 & 9.52 & 10. 14 & 10. 81 & 10. 7 & 10.63 & 10. 31 \\
\hline
\end{tabular}

\section{Analysis of the Relationship between Urbanization and Education Level in Hainan Province}

This paper analyzes the impact of urbanization on education, and sets the level of urbanization as $\mathrm{X}$ and the educational level Y. Hainan Province, 18 cities and counties in 2000 and 2011 is processed by software Eviews6.0 data, $\mathrm{Y}$ and $\mathrm{X}$ are carried out unit root test, using Levin, Lin \& Chu $\mathrm{t} *$ and ADF - Fisher Chi-square two test standards, the test found $\mathrm{Y}$ is the first order single product, $\mathrm{X}$ is also the 
first order single whole, respectively, $\mathrm{Y}$ and $\mathrm{X}$ first seeking logarithm, and then take the first step after the smooth, through cointegration test, finds that direct regression analysis, the results (table 4).

Table 4 urbanization and education take the first-order difference after the regression results

\begin{tabular}{|c|c|c|c|c|}
\hline Variable & Coefficient & Std. Error & t-Statistic & Prob. \\
\hline C & 0.004331 & 0.001066 & 4.064509 & 0.0001 \\
\hline DLNX? & -0.03101 & 0.00873 & -3.551983 & 0.0005 \\
\hline R-squared & 0.060477 & \multicolumn{2}{|c|}{ Mean dependent var } & 0.002398 \\
\hline Adjusted R-squared & 0.055684 & \multicolumn{2}{|c|}{ S.D. dependent var } & 0.013265 \\
\hline S.E. of regression & 0.01289 & \multicolumn{2}{|c|}{ Akaike info criterion } & -5.854638 \\
\hline Sum squared resid & 0.032567 & \multicolumn{2}{|c|}{ Schwarz criterion } & -5.821423 \\
\hline Log likelihood & 581.6091 & \multicolumn{2}{|c|}{ Hannan-Quinn criter. } & -5.841193 \\
\hline F-statistic & 12.61658 & \multicolumn{2}{|c|}{ Durbin-Watson stat } & 1.977226 \\
\hline Prob(F-statistic) & 0.000479 & & \\
\hline
\end{tabular}

Finally, the result is that the relationship between the educational level $\mathrm{Y}$ and the urbanization level $\mathrm{X}$ is expressed as (three digits after the decimal point):

$$
\ln \mathrm{Y}=0.004-0.031 * \ln \mathrm{X}
$$

The results show that the impact of urbanization on the level of education is negative for the negative factors, but careful observation of the level of education in Hainan Province and urbanization data found that data in Hainan Province, there are data changes. Therefore, after a phased analysis, it was found that the relationship between education level and urbanization level in Hainan Province from 2003 to 2008 (Table 5) was consistent with common sense.

Table 5 urbanization and education take the first-order difference after the regression results

\begin{tabular}{|c|c|c|c|c|}
\hline Variable & Coefficient & Std. Error & t-Statistic & Prob. \\
\hline C & 0.006639 & 0.001165 & 5.697987 & 0 \\
\hline DLNX? & 0.02055 & 0.010297 & 1.99577 & 0.0499 \\
\hline R-squared & 0.053838 & \multicolumn{2}{|c|}{ Mean dependent var } & 0.00783 \\
\hline Adjusted R-squared & 0.040321 & \multicolumn{2}{|c|}{ S.D. dependent var } & 0.008669 \\
\hline S.E. of regression & 0.008492 & \multicolumn{2}{|c|}{ Akaike info criterion } & -6.671998 \\
\hline Sum squared resid & 0.005048 & \multicolumn{2}{|c|}{ Schwarz criterion } & -6.608757 \\
\hline Log likelihood & 242.1919 & Hannan-Quinn criter. & -6.646821 \\
\hline F-statistic & 3.9831 & Durbin-Watson stat & 2.195571 \\
\hline Prob(F-statistic) & 0.049851 & & \\
\hline
\end{tabular}

The result is that the relationship between the level of education $\mathrm{Y}$ and the level of urbanization $\mathrm{X}$ is expressed as:

$$
\ln \mathrm{Y}=0.006+0.021 * \ln \mathrm{X}
$$

(5) represents the economic meaning of the level of urbanization for each $1 \%$ increase in the number of education will increase by $0.021 \%$. While the other two stages, namely, 2000-2002, $2009-2011$, the relationship between the two were:

$$
\operatorname{LnY}=-0.013-0.122 * \ln \mathrm{X}(6) \ln \mathrm{Y}=-0.051 * \ln \mathrm{X}
$$

Therefore, the impact of urbanization in Hainan Province on the level of education in 2000-2011, the phased to analyze, in 2000-2002 and 2009 - 2011 two stages, Hainan Province, the level of urbanization and education between the existence of And there is a positive correlation between the urbanization level and the educational level in Hainan Province during the period of 2003-2008. The reason for this phenomenon is due to the fact that Hainan Province is in the stage of urbanization and the development of education level. There are still some gaps in the initial stage, and there are other non-normal factors leading to the decline of the educated population and the shortened period of education. However, it is undeniable that the urbanization level and educational level of 18 cities and counties in Hainan Province In 2000 and 2011 are on the overall growth trend, and the impact of urbanization on the level of education in most of the time is a positive impact, and in the future must also be a positive impact. 


\section{Conclusion Analysis and Related Discussion}

Based on the statistical data of 18 cities and counties in Hainan Province from 2000 to 2011, this paper analyzes the relationship between urbanization and education level in Hainan Province based on structural equation model and Eviews software, and we draw the following conclusions and discussion:

1, On the whole, the pace of development of urbanization in Hainan Province is slower than that of the whole country. However, the urbanization level of 18 cities and counties has been improved in the past ten years. The process of urbanization has been continuously promoted. Population urbanization, economic urbanization and social urbanization have also become measures of improving the level of urbanization, the main indicators and influencing factors. And it can be clearly found that the impact factors of urbanization, economic urbanization and social urbanization on the level of urbanization are quite large, especially the urbanization of population, and the overall urbanization level of Hainan Province Low, so in the context of the development of the real estate economy and international tourism island, Hainan Province should accelerate the pace of urbanization and promote the urbanization of economy and society in order to meet the level of urbanization Material and living needs.

2, Through the software Eviews6.0, we can conclude that the level of urbanization on the education level of a greater impact, although some years due to specific reasons for the abnormal situation, but in general, the level of urbanization and education level is in the stage of accelerating the growth, which is bound to the future trend. The impact of urbanization on the level of education is positive, the two are positive correlation, but because of the low level of social and economic development in Hainan Province, multi-ethnic and rural population, social awareness and education awareness is not particularly strong, A period of decline or abnormal phenomenon should be normal. This also shows that the Hainan provincial government needs to accelerate the pace of urbanization, while promoting the urbanization and education of the far-reaching significance, improve people's awareness and quality, for the level of urbanization and education to create conditions for improvement.

3, Hainan Province is located in the southernmost part of China, economic development in the geographical conditions on the obvious advantages, but other conditions (such as education, science and technology, talent and other aspects) are still big disadvantages, and the population is relatively small, generally low level of education, government should increase the level of urbanization and education to improve the level of financial investment, while universal urbanization and the level of education to improve the benefits to promote and promote development.

4, In this paper, when the structural equation model is analyzed, the time span and comprehensiveness of the data are not enough. Because of the limitation of the statistical yearbook data, this paper chooses the population, economy and society to analyze the urbanization, and the regression analysis, the time span is also slightly short, and there is a negative correlation between the urbanization and the education level in the study results, indicating that the actual situation of 18 cities and counties in Hainan Province is acceptable, and urbanization shall be an inevitable trend, which is also reflected in the 2003-2008 obvious.

\section{References}

[1] Journal of Urban Development Research, 2012, 4: 020 (in Chinese with English abstract) [J]. Journal of Urban Development Research, 2012, 4: 020.

[2] Xu Chaojun, Luo Nengsheng, Wang Jiaqing.The Promotion of Education to Urbanization [J]. Journal of Economic Geography, 2007, 27 (4): 607-609.

[3] China Population and Development Research Center Task Force. China's population and education development strategy research [J]. Population Research .2009, 3: 4-19.

[4] Wang Jia-ting, Cui Feng-yu. Empirical Study on the Relationship between Population Education Structure and Urbanization in China [J]. Urban Development Research, 2010, 17 (12): 24-29. 
[5] Zhang Xinmin. An Empirical Study on the Impact of Urbanization on Rural Education Development - Based on Panel Data Analysis in Henan Province [J]. Statistics and Decision, 2011, (5): 105-107.

[6] Weng Jinghua, Han Yuqi. Evaluation of urbanization and higher education [J]. Urban Development Research, 2012, 19 (5): inserted in 1 - inserted 3, inserted 8.

[7] Chen Yongjun: China's urbanization development practice of a number of theoretical and policy issues [J]. Economics .2010 (1): 25-31.

[8] Liao Yi, Tang Yufeng. Empirical Study on the Contribution of Human Capital to Hunan's Economic Growth [J]. Economic Geography, 2012, (1): 41-45, 95. 